\title{
Efficacy and safety data of belimumab in patients with systemic lupus erythematosus
}

\author{
Bita Shakoory and Winn Clatham
}

\begin{abstract}
Belimumab is a human genome derived monoclonal antibody with specificity for BLyS (B lymphocyte stimulator, or B-cell activating factor [BAFF]), a cytokine that promotes the survival and maturation of $\mathrm{B}$ cells into antibody-secreting plasmablasts. Recent phase III clinical trials with belimumab in patients with active systemic lupus erythematosus (SLE) have been completed and achieved primary efficacy endpoints employing validated disease activity measures (SLEDAI and BILAG) as well as additional secondary endpoints related to disease flares and sparing of corticosteroid use.

Significant decreases in numbers of activated B cells as well as levels of autoantibodies are observed during treatment with belimumab. The majority of observed clinical improvements are observed in musculoskeletal, mucocutaneous, and serologic domains of disease activity; the potential effects on more severe neurologic or renal domains of disease are not known. Treatment with belimumab is well tolerated and has not been associated with significant toxicity.
\end{abstract}

Keywords: lupus, BLyS, BAFF, belimumab, autoantibodies, B-lymphocytes

\section{Background}

Systemic lupus erythematosus (SLE) is an autoimmune disorder associated with accentuated activation of B lymphocytes and multiple organ system manifestations. The disease mainly affects women in their reproductive years with significant morbidity and impact upon the quality of life of affected patients [Lau and Mak, 2009; D'Cruz et al. 2007]. However, until its recent approval no new treatment had been approved by the US Food and Drug Administration for use in SLE since the approval of hydroxychloroquine in 1958.

The heterogeneity of SLE with respect to clinical manifestations, immunopathogenetic pathways as well as the burden of comorbidities have posed significant challenges to the identification of appropriate therapies and confirmation of their clinical efficacy [Schröder and Zeuner, 2009]. Recent insights into the biology of B cells and the immunobiology of SLE have lead to the development of potential new therapies targeting B lymphocytes in patients with SLE. B Lymphocyte Stimulator (BLyS, also referred to as $\mathrm{B}$-cell activating factor [BAFF]) is a tumor necrosis factor (TNF) family ligand for two known receptors on $\mathrm{B}$ cells (BAFF-R and TACI) that mediate the survival of $B$ cells and their differentiation into plasmablasts [Cancro et al. 2009; Moore et al. 1999]. Elevated levels of BLyS/BAFF have been demonstrated in patients with SLE and targeting of BLyS/BAFF in murine models of lupus results in significant amelioration of murine disease [Liu et al. 2004; Zhang et al. 2001].

Belimumab is a recombinant human genome derived IgG1 $\lambda$ monoclonal antibody with specificity for soluble (nonmembrane bound) BLyS [Baker et al. 2003]. Belimumab was found to have biologic activity and a favorable safety profile in phase I and II studies and is the first of these potential new therapies to have met the primary efficacy endpoints in phase III clinical trials [Petri et al. 2010; Navarra et al. 2009]. The phase III belimumab studies included a large cohort of 865 enrolled/treated subjects in Asia, South America and Eastern Europe (BLISS-52), and a similar sized cohort of 819 enrolled/treated subjects in North America and Western Europe (BLISS-76) randomized equally to receive either placebo, $1 \mathrm{mg} / \mathrm{kg}$, or $10 \mathrm{mg} / \mathrm{kg}$ treatment with belimumab every 4 weeks. In this overview
Ther Adv Musculoskel Dis (2011) 3(3) 159-164 DOI: $10.1177 /$ $1759720 \times 11407541$

(C) The Author(s), 2011. Reprints and permissions: http://www.sagepub.co.uk/ journalsPermissions.nav

Correspondence to: Dr Winn Clatham, MD University of Alabama at Birmingham, Clinical Immunology and Rheumatology/Medicine, Birmingham, AL, USA Winn.Chathama ccc.uab.edu

Bita Shakoory, MD University of Alabama at Birmingham, Clinical Immunology and Rheumatology/Medicine, Birmingham, AL, USA 
we focus on the immunologic effects, clinical efficacy, and safety of belimumab observed in the phase II and III clinical trials.

\section{Efficacy}

\section{Biologic activity and biomarkers}

$B$-cell and T-cell subsets. As observed in the phase II study with belimumab [Wallace et al. 2009] and confirmed in the subsequent phase III trials [Stohl et al. 2010], significant decreases in the measured numbers of circulating activated $\mathrm{B}$ cells and plasmacytoid B lymphocytes were observed in belimumab treatment groups compared with placebo groups. A transient increase in the number of circulating memory $B$ cells is observed immediately following administration of belimumab, with these numbers gradually returning to the pretreatment baseline level over the course of several months of treatment. Total numbers of circulating B cells were decreased $20-25 \%$ over the 1 -year treatment periods in the respective trials, with no observed decreases in CD4 and CD8 T lymphocytes.

Autoantibodies and immunoglobulins. Early and statistically significant reduction in autoantibodies including anti-ds-DNA, anti-Smith, anticardiolipin G and anti-RNP antibodies were demonstrated in belimumab-treated groups compared with the placebo groups in the phase II and III studies [Stohl et al. 2010; Wallace et al. 2009]. The noted $40-50 \%$ decreases in levels of autoantibodies has been sustained in the phase II trial cohort of patients remaining in the open-label $(10 \mathrm{mg} / \mathrm{kg}$ monthly dosing) long-term extension study, with continued decrease in the titers of these autoantibodies having been observed over 5 years of observation [Merrill et al. 2010]. Serum levels of IgG were observed to decrease by an average of $15 \%$ in the belimumab-treated groups over the 1-year course of treatment [Stohl et al. 2010]; further decreases have not been observed in the open-label cohort extending out to 5 years of treatment. For subjects who had received immunization to influenza, tetanus toxoid or pneumococcus prior to study entry, there were no significant decreases in the titers of antibodies to influenza, tetanus or pneumococcal antigens observed over the course of treatment during the phase III study [Chatham et al. 2010]. These observations suggest that treatment with belimumab may have a greater impact on autoreactive B cells than on lymphoid lineage cells required for maintaining humoral host defense.

Serum complement. Significant increases in the serum levels of complement (C3, C4) were also observed to increase in the belimumab treatment groups compared with placebo groups during the course of the phase II and III studies, with significantly greater numbers of subjects in the belimumab groups achieving normalization of previously decreased C3 and C4 levels by week 52 in both of the phase III trials [Stohl et al. 2010; Wallace et al. 2009]. These observed increases and normalization in serum complement levels were confirmed at week 76 in the BLISS-76 trial and have been sustained among subjects remaining in the phase II long-term extension cohort [Merrill et al. 2010].

\section{Clinical efficacy}

Owing to the heterogeneity of disease manifestations and uncertainties with regard to the temporal onset of biologic activity sufficient to impact clinical outcomes, the assessment of clinical efficacy for interventions in SLE is a major challenge. The disease activity measures currently employed to define efficacy endpoints in most SLE trials include the SLE Disease Activity Index (SLEDAI) and/or British Isles Lupus Assessment Group (BILAG) index. In the phase II trial with belimumab, none of the belimumab treatment groups achieved a statistically significant improvement in predetermined endpoint of a four-point improvement in the SLEDAI score. However, in a post hoc analysis of the phase II experience, applying a combined SLE response index (SRI) of a four-point improvement of the SLEDAI score with no worsening of any domains on the BILAG and no worsening of the Physician Global Assessment (PGA) score to subjects who had confirmed elevated antinuclear antibody (ANA) or antidsDNA at study entry demonstrated significantly greater numbers of belimumab-treated than placebo-treated subjects in this subset achieved the CRI [Furie et al. 2009]. Noteworthy clinical correlates of the ANA and/or anti-dsDNA positive subset of subjects in this analysis included African American race, younger age, major organ involvement, higher prednisone use, lower complement levels, higher immunoglobulin levels, higher SELENA-SLEDAI score at baseline and higher frequency of detectable BLyS levels. 
The SRI derived from the belimumab phase II experience was employed as the primary endpoint in both of the phase III studies. In these trials, stable background therapy with antimalarials or immunosuppressant medications including methotrexate, azathioprine or mycophenolate was permitted (although not required) as well as stable use of corticosteroids in the month preceding study entry. Study subjects were required to have a SLEDAI score of at least six to enter both trials; the mean SLEDAI score across all treatment groups at study entry was 9.6, with scored disease activity on the SLEDAI predominantly in the mucocutaneous and musculoskeletal domains [Manzi et al. 2010]. Patients with severe renal or neuropsychiatric manifestations were notably excluded from entry into the phase III trials.

Both of the phase III studies met the primary efficacy endpoint as a greater percentage of subjects treated with belimumab $(10 \mathrm{mg} / \mathrm{kg}$ at 4 -week intervals) compared with placebo achieved the SRI at 52 weeks in the BLISS-52 as well as the BLISS-76 study [Furie et al. 2010; Navarra et al. 2009]. The percentage of subjects meeting the SRI at week 76 in the phase III BLISS-76 study (a predefined secondary endpoint) was greater in the belimumab-treated groups relative to placebo but the difference failed to achieve statistical significance; however, when applying more stringent criteria of five-, six-, or seven-point decreases in the SLEDAI component of the SRI, significantly greater percentages of subjects in the $10 \mathrm{mg} / \mathrm{kg}$ belimumabtreated group relative to the placebo group $(p<0.05)$ achieved these benchmarks of clinical improvement at week 76 as well as at week 52 [Furie et al. 2010]. In both of the phase III trials, improvements in musculoskeletal, mucocutaneous, and serologic domains accounted for the majority of noted improvements in the SLEDAI and BILAG [Manzi et al. 2010].

An additional secondary endpoint in the phase II and III studies included reduction in disease flares as defined by the SLEDAI flare index. Reduction in the numbers of severe flares (20.4\% in placebo group and $15.2 \%$ in the belimumab group) did not achieved statistical significance in the phase II study $(p=0.20)$; however, when the start point of analysis for flares is moved to week 24, a significant difference in the time to first flare was apparent (154 days in the belimumab groups and 108 days in the placebo group, $p=0.0361$ ) [Wallace et al. 2009]. Data presented from the two large phase III trials confirms a decreased frequency of flares in the belimumabtreated groups during the second 6 months of treatment and suggests an apparent diseasestabilizing attribute of belimumab [Petri et al. 2010]. Although the cohort in the 5-year openlabel follow-up study is likely enriched for responders, both the overall rate of SLEDAI flare and the severe flare index have consistently declined for patients that remain in the cohort [Merrill et al. 2010]. These observations and the delays in maximal clinical responses noted in the respective outcome measures are not unexpected in the context of belimumab's mechanism of action: targeting a growth and survival factor for B cells would not be expected to have a significant clinical impact until several months following initiation of treatment, but the noted improvements following this time interval might well be sustained over time.

Reduction in corticosteroids was also evaluated as a secondary endpoint in the phase II and III studies with belimumab. In the phase II study, a significantly lower proportion of the low-prednisone users or nonusers in the belimumab $10 \mathrm{mg} / \mathrm{kg}$ group compared with the placebo group had increases in their prednisone to $>7.5 \mathrm{mg}$ daily during the course of the study. Furthermore, a greater number of subjects entering the trial on $>7.5 \mathrm{mg} /$ day prednisone in the belimumab $10 \mathrm{mg} / \mathrm{kg}$ group experienced significant reductions in daily prednisone use $(50 \%$ dose decrease to $<7.5 \mathrm{mg} /$ day) in the final 3 months of the study compared with the placebo group; however, the difference failed to achieve statistical significance [Wallace et al. 2009]. In the two phase III trials, a greater percentage of belimumab-treated subjects reached a similar secondary endpoint $(25 \%$ reduction in daily prednisone to $<7.5 \mathrm{mg}$ /day at week 52 ) relative to the placebo group although the noted differences achieved statistical significance only in the BLISS-52 study [Furie et al. 2010; Navarra et al. 2009]; notably, the average dose of corticosteroids for subjects entering the trial was also significantly greater in the BLISS-52 study population (with greater use of nonsteroid immunosuppressive therapy prevalent in the BLISS-76 study population). In a combined analyses of the two phase III study groups, significantly greater numbers of subjects in the $10 \mathrm{mg} / \mathrm{kg}$ belimumabtreated group were able to lower their use of prednisone to $<7.5 \mathrm{mg} /$ day, and significantly 
fewer numbers of subjects in this group required increases in their prednisone use over this threshold [Petri et al. 2010]. Other potential measures of steroid sparing effect such as cumulative exposure over the course of the study or segments thereof have not been reported.

Secondary patient-reported outcome measures of interest in the phase III trials included fatigue as measured by the FACIT-fatigue scale and healthrelated quality of life outcomes as measured by the Short Form 36 (SF-36) Physical Component Summary (PCS). Greater absolute changes in the FACIT-fatigue score were noted in both of the belimumab-treated groups relative to the placebo group in the BLISS-52 study [Navarra et al. 2009]; similar greater improvements in fatigue scores in the belimumab-treated groups were noted in the BLISS-76 study although the noted improvements did not attain statistical significance in the $10 \mathrm{mg} / \mathrm{kg}$ treatment group [Furie et al. 2010]. Score improvements on the SF-36 PCS were significantly greater at week 52 for the belimumab-treated groups relative to placebo in the BLISS-52 trial [Navarra et al. 2009]; with the exception of the $1 \mathrm{mg} / \mathrm{kg}$ group at week 52 , improvements observed in the belimumab-treated groups in the BLISS-76 study did not reach statistical significance relative to the placebo group at week 52 or week 76 [Furie et al. 2010].

\section{Safety and tolerability}

The experience with belimumab in the phase I, II, and III trials indicates the reagent is generally safe and well tolerated with comparable frequency of adverse events (AEs) and serious adverse events (SAEs), including infections and serious infections across placebo and treatment groups. In the phase I study, only one of the patients in the belimumab group experienced infusion interruption due to urticaria and chest pain, requiring histamine blockade [Furie et al. 2008]. Except for an infusion-site reaction in one patient and more infusion-related urticaria events ( $4 \%$ belimumab groups versus $0 \%$ in placebo subjects), belimumab was well tolerated in the phase II study [Wallace et al. 2009]. The notable SAEs in the phase II study are two malignancies, one severe infusion reaction and two deaths. Excepting the infusion reaction, none of these incidents (one suicide, one death related to respiratory failure, one basal cell carcinoma and one squamous cell carcinoma) were attributed to the study agent by the respective principal investigators. In the 5-year open-label extension of the phase II study, the reported discontinuation rate has been 3-9\% per year with none of the drop outs attributable to the side effects of belimumab [Merrill et al. 2010]. A total of 23 incidents of malignancy have been reported with 1415 patient-years exposure to belimumab; these have been evenly distributed during the 5-year period of follow up.

The overall favorable tolerability and safety profile of belimumab was confirmed in both of the larger phase III studies, where infusion-related events and discontinuation of the study drug due to adverse events were comparable across belimumab-treated and placebo groups [Wallace et al. 2010]. No statistically significant differences were observed in the prevalence of adverse events, serious adverse events, infection-related AEs or SAEs, or deaths across belimumab-treated and placebo-treated subject groups. Malignancies were reported in four patients in the phase III studies, three of whom were in the belimumab-treated groups and one of whom was in the placebo group [Wallace et al. 2010].

Given the role of BLyS/BAFF in promoting $\mathrm{B}$-cell survival through ligation of BAFF-R as well as being one of the two known ligands (the other being APRIL) impacting immunoglobulin class switch through ligation of TACI, the levels of serum immunoglobulin in subjects treated with belimumab over time is of interest. In the phase III trials, decreases in total IgG (15-16\%), IgA (18-20\%), and $\operatorname{IgM}(32-35 \%)$ were observed in the belimumab-treated groups; in the BLISS-76 study only one subject in each of the belimumab and placebo groups developed hypogammaglobulinemia [Stohl et al. 2010]. None of the subjects in the phase II open-label long-term extension study have been reported to have developed hypogammaglobulinemia [Merrill et al. 2010]. Although antibody titers to pneumococcal, influenza, and tetanus antigens among immunized subjects entering the BLISS76 trial did not decrease over the course of treatment with belimumab, it is not known at this point to what, if any, extent treatment with belimumab impacts primary immunization responses [Chatham et al. 2010].

\section{Summary}

Results from clinical trials completed thus far suggest that when added to standard of care treatment, belimumab is a safe and moderately effective treatment for patients with SLE, and it may 
have a role in decreasing corticosteroid requirements for disease management. Improvement in serologic abnormalities, musculoskeletal, and mucocutaneous disease manifestations comprise the majority of demonstrated improvements observed thus far in response to treatment with belimumab; the onset of clinical benefit is relatively slow, perhaps not surprising given its mechanism of action. Relative to the $1 \mathrm{mg} / \mathrm{kg}$ belimumab treatment group, the majority of improved primary and secondary outcomes in the phase III trials were observed in the $10 \mathrm{mg} /$ $\mathrm{kg}$ belimumab treatment groups.

The extent to which belimumab may be of benefit in the management of patients with more severe renal or neurologic manifestations is not yet known. Going forward, it will be of interest to determine whether belimumab has a beneficial role in preventing relapses of these more severe manifestations once they have remitted in response to conventional less targeted therapies such as cyclophosphamide, azathioprine, mycophenolate and/or high-dose corticosteroids. Additional studies making use of genomic and/ or proteonomic biomarkers are also needed and will hopefully be useful to determine which subpopulations of patients with SLE are most likely to benefit from this new and novel intervention.

\section{Funding}

This research received no specific grant from any funding agency in the public, commercial, or notfor-profit sectors.

\section{Conflict of interest statement}

W. Winn Chatham has received research or grant support and travel support from Human Genome Sciences as an investigator in the preclinical studies, phase I, phase II, and phase III clinical trials with belimumab.

\section{References}

Baker, K.P., Edwards, B.M., Main, S.H., Choi, G.H., Wager, R.E., Halpern, W.G. et al. (2003) Generation and characterization of LymphoStat-B, a human monoclonal anti-body that antagonizes the bioactivities of B lymphocyte stimulator. Arthritis Rheum 48: 3253-3265.

Cancro, M.P., D’Cruz, D.P. and Khamashta, M.A. (2009) The role of B lymphocyte stimulator (BLyS) in systemic lupus erythematosus. $\mathcal{F}$ Clin Invest 119: 1066-1073.
Chatham, W.W., Wallace, D.J., Stohl, W., Latinis, K., Manzi, S., McCune, J. et al. (2010) Effect of belimumab, a B-lymphocyte stimulator specific inhibitor, on functional antibodies to pneumococcal, tetanus, and influenza vaccines. Arthritis Rheum 62(Suppl. 10): 457.

D'Cruz, D.P., Khamashta, M.A. and Hughes, G.R. (2007) Systemic lupus erythematosus. Lancet 369: 587-596.

Furie, R., Stohl, W., Ginzler, E., Becker, M., Mishra, N., Chatham, W. et al. (2008) Biologic activity and safety of belimumab, a neutralizing anti-B-lymphocyte stimulator (BLyS) monoclonal antibody: a phase I trial in patients with systemic lupus erythematosus. Arthritis Res Ther 10: R109.

Furie, R., Zamani, O., Wallace, D., Tegzová, D., Petri, M., Merrill, J.T. et al. (2010) Belimumab, a BLyS-specific inhibitor, reduced disease activity and severe flares in seropositive SLE patients: BLISS-76 study results through Wk 76. Arthritis Rheum 62(Suppl. 10): 606.

Furie, R.A., Petri, M.A., Wallace, D.J., Ginzler, E.M., Merrill, J.T., Stohl, W. et al. (2009) Novel evidencebased systemic lupus erythematosus responder index. Arthritis Rheum 61: 1143-1151.

Lau, C.S. and Mak, A. (2009) The socioeconomic burden of SLE. Nat Rev Rheumatol 5: 400-404.

Liu, W., Szalai, A., Zhao, L., Liu, D., Martin, F., Kimberly, R.P. et al. (2004) Control of spontaneous B lymphocyte autoimmunity with adenovirus-encoded soluble TACI. Arthritis Rheum 50: 1884-1896.

Manzi, S., Sanchez-Guerrero, J., Merrill, J.T., Furie, R.A., Gladman, D., Navarra, S. et al. (2010)

Belimumab, a BLyS-specific inhibitor, reduced disease activity across multiple organ domains: combined efficacy results from the phase 3 BLISS-52 and -76 studies. Arthritis Rheum 62(Suppl. 10): 1456.

Merrill, J.T., Wallace, D.J., Furie, R.A., Petri, M.A., Stohl, W., Chatham, W.W. et al. (2010) Five-year experience with belimumab, a BLyS-specific inhibitor, in patients with systemic lupus erythematosus (SLE). Arthritis Rheum 62(Suppl. 10): 1457.

Moore, P.A., Belvedere, O., Orr, A., Pieri, K., LaFleur, D.W., Feng, P. et al. (1999) BLyS: member of the tumor necrosis factor family and B lymphocyte stimulator. Science 285: 260-263.

Navarra, S., Guzman, R., Gallacher, A., Levy, R.A., Li, E.K., Thomas, M., et al. (2009) Belimumab, a BLyS-specific inhibitor, reduced disease activity, flares and prednisone use in patients with active SLE: efficacy and safety results from the phase 3 BLISS-52 study. Arthritis Rheum 60: 38593861.

Petri, M.A., Levy, R.A., Merrill, J.T., Navarra, S., Cervera, R., van Vollenhoven, R.F. et al. (2010)

Belimumab, a BLyS-specific inhibitor, reduced disease 
Visit SAGE journals online http://tab.sagepub.com

(SSAGEJOURNALS Online activity, flares, and prednisone use in patients with seropositive SLE: combined efficacy results from the phase 3 BLISS-52 and -76 studies. Arthritis Rheum 62(Suppl. 10): 452 .

Schröder, J.O. and Zeuner, R.A. (2009) Biologics as treatment for systemic lupus: great efforts, sobering results, new challenges. Curr Drug Discovery Technol 6: 252-255.

Stohl, W., Hiepe, F., Thomas, M., Scheinberg, M.A., Clarke, A.E., Chatham, W. et al. (2010) Belimumab, a BLyS-specific Inhibitor, significantly reduced autoantibodies, normalized low complement, and reduced selected B-cell populations in patients with seropositive systemic lupus erythematosus (SLE): The Phase 3 BLISS studies. Arthritis Rheum 62(Suppl. 10): 1146.
Wallace, D.J., Navarra, S., Gallacher, A., Guzman, R., Thomas, M., Furie, R.A. et al. (2010) Safety Profile of Belimumab, a BLyS-specific inhibitor, in patients with active systemic lupus erythematosus (SLE): pooled data from phase 2 and 3 studies. Arthritis Rheum 62(Suppl. 10): 1172.

Wallace, D.J., Stohl, W., Furie, R.A., Lisse, J.R., McKay, J.D., Merrill, J.T. et al. (2009) A phase II, randomized, double-blind, placebo-controlled, doseranging study of belimumab in patients with active systemic lupus erythematosus. Arthritis Rheum 61: 1168-1178.

Zhang, J., Roschke, V., Baker, K.P., Wang, Z., Alarcon, G.S., Fessler, B.J. et al. (2001) Cutting edge: a role for B lymphocyte stimulator in systemic lupus erythematosus. F Immunol 166: 6-10. 